\title{
Real-Time Monitoring System of Microseismic Signal based on Virtual In- strument and Wavelet Analysis
}

\author{
Lu Zeng ${ }^{1, *}$, Rongshuang $\mathrm{Lu}^{1}$ and Qiang $\mathrm{Wen}^{2}$ \\ ${ }^{1}$ School of Electrical Engineering and Automation, Jiangxi University of Science and Technology, GanZhou, 341000, \\ JiangXi, \\ ${ }^{2}$ School of Electronic and Information Engineering, South China University of Technology, Guangzhou, 510000, \\ Guangdong
}

\begin{abstract}
Seismic acquisition and monitoring system can collect, store, analyze and dispose the rock burst seismic waves or the vibration of other objects. This paper utilizes single chip computer technology, virtual instrument technology, sensor technology, database management technology, wavelet analysis technology, combining the real condition of the mine microseismic signal to establish a set of mine microseismic signal real time monitoring and warning system. Finish the seismic signals data collection by the single chip microcomputer and its periphery circuit design, meanwhile use the powerful graphical programming capabilities and flexible diversify data processing functions of the LabVIEW software, combining with advanced digital signal processing technology such as wavelet transform to complete seismic signal acquisition and filtering processing, records and analysis, monitoring and early warning functions which offers evidence for the safety monitoring of underground mine and has a good application prospect.
\end{abstract}

Keywords: Microseismic signal, Data collection, Virtual Instrument, Wavelet de-noising.

\section{INTRODUCTION}

Seismic acquisition and monitoring system can be generated for rock burst of the vibration of the seismic waves, or other objects for collection storage, analysis and processing. Which can confirm the scope and degree of underground rock failure, so as to prevent the happening of the disaster. however, the cost of the existing mine geological safety monitoring instruments is high, and most function is single and so, difficult to operate, decorate in the large number of mining area. How to lower the cost to design efficient stable for monitoring solution has been an urgent demand $[1,2]$.

In the face of this, this paper has studied and developed a micro-seismic monitoring system, which is efficient, stable, intelligent, easy operation, economical and pratical, and based on virtual instrument. In order to reduce the system cost, the system give up using PCI acquisition card scheme, and choose the hypogyny machine with the single chip processor as the core of the data acquisition module on the rock burst of vibration signal in real time collection, The module includes seismic detector, amplifier circuit, filter circuit, AD digital to analog conversion circuit and single chip microcomputer control system. The System introduced the LabVIEW software platform, which could intuitive display, analysis, storage and handling with visual way, and improved the ability of man-machine interaction. At the same time, utilizing the powerful data processing ability of MATLAB and the advanced wavelet algorithm optimize the collected signals, which could improve detection precision and efficiency to the greatest degree. So as to improve the safety factor of mine development, reduce the economic loss caused by the mine and the casualties [3-5].

\section{SYSTEM OVERALL STRUCURE DESIGN}

\subsection{The System Overall Design}

The microseismic monitoring system includes two parts of the hardware design and software design.

The traditional microseismic monitoring schemes mostly use NI company provide PCI acquisition card to collect microtremor signals, but this kind of peripherals cost is higher, is not conducive to a wide range of use, in order to reduce the hardware cost, this solution to AT89C52 single chip microcomputer as the core of data acquisition system to complete the acquisition process, including seismic detection, signal amplifier, a low-pass filter, analog-to-digital conversion, the RS-232 communication circuit, single chip microcomputer system board and so on $[6,7]$.

The block diagram of hardware system is roughly as Fig. (1).

The part of software uses LabVIEW development platform to implement the following functions:

1. VISA to send and receive serial data. 


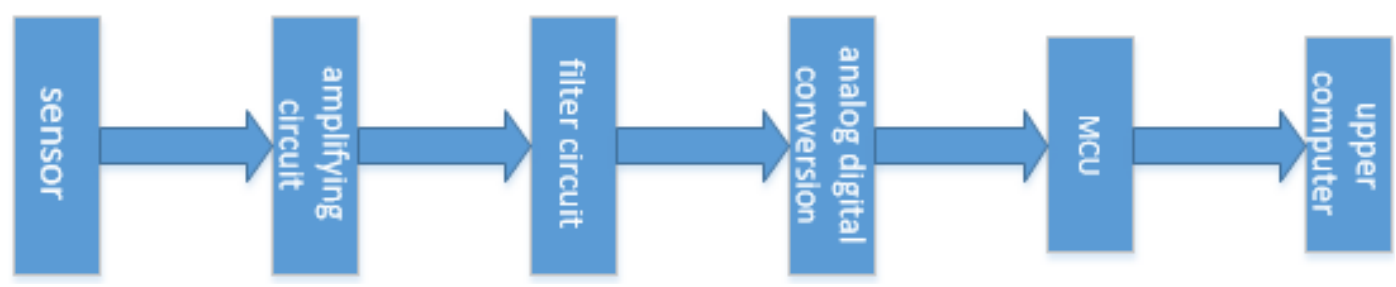

Fig. (1). The system hardware design.

2. Four-channel real-time waveform display and channel opening and closing is optional.

3. Using the wavelet de-noising algorithm.

4. Waveform could storage, playback and keep the waveform for pictures.

5. Designing a landing window for the system, and log in to use this system correctly.

\subsection{The Hardware System Design}

\subsubsection{Signal Conditioning Circuit Design}

Microtremor signals were collected by seismic geophone, which cannot be directly used for experimental analysis, to reach the requirements of digital to analog conversion to input level after circuit needs a series of signal conditioning circuit, the signal conditioning module includes amplifying circuit, filter circuit, etc.

\section{$>$ The front-end amplifier circuit}

The front-end amplifier circuit design is shown in Fig. (3)

Microseismic signal signal amplitude is small, the electrical signals is converted by sensor, which cannot make modulus conversion directly. So, it needs to enlarge the original signal. However, because of the original signal with a lot of noise, the first level amplification processing is particularly important, in order to restrain noise interference, the frontend amplifier usually adopts differential input method, the circuit need to meet the following features:

The amplifying circuit with high input impedance, in order to reduce the error which is introduced by the signal source resistance does not match.

With high common mode to suppress ratio, to reduce the error of the common-mode noise introduced.

The amplifying circuit with high gain, and gain the adjusting range is wide, to adapt to the scope of the signal level.

Based on the above requirements, the system adopts AD8574 four-channel operational amplifier as a front-end amplifier circuit of the op-amp. The AD8574 chip can provide the unique characteristic advantage which only expensive steady stable zero or chopper amplifier possess. Using the topology of the ADI company, the zero drift amplifier will combine low cost and high precision characteristics, and without external capacitance. Making use of spread spectrum, the stability of zero patent technology, which can eliminate communication applications chopped wave function and the signal frequency tuning effect caused by the interaction. The chip has the following features:
$>$ High gain and common mode rejection ratio. AD8574 single channel gain can reach $145 \mathrm{db}$, common mode rejection ratio can reach $140 \mathrm{db}$.

$>\quad 5 \mathrm{~V} / 2.7 \mathrm{~V}$ single power supply, can simplify the hardware circuit.

$>$ Low offset voltage is only $1 \mu \mathrm{V}$, input offset drift just $0.005 \mu \mathrm{V} /{ }^{\circ} \mathrm{C}$

$>$ Without external capacitance.

$>$ Multi-channel, there are four high accuracy operational amplifiers; a single chip can provide the first and the end of the signal amplifying and filtering circuit Fig. (2) is the AD 8574 pins.

Circuit magnification

$A u=-\left(\frac{R 5}{R 6}+1\right)=-10$

This circuit can provide ten times of original signal amplifier, amplified signal is access after implantation of big filter circuit, $300 \mathrm{~Hz}$ filtering and second-level amplification [8].

Active low-pass filter circuit

Due to seismic signals is low frequency signal, its frequency ranges about $20 \mathrm{hz}$ to $300 \mathrm{hz}$, in view of the low frequency characteristics of seismic signal, the system sets the second-order active low-pass filter cutoff frequency is 400 hz. And finishs about 2.5 times of the signal amplification.

This system adopts the typical second-order butterworth low-pass filter, the feature of butterworth filter passband is the frequency response curve of maximum flat, no ups and downs, and the resistance band is gradually reduced to zero, the second order butterworth filter attenuation rate for each frequency doubling $12 \mathrm{db}$. Filter the op-amp still uses AD8574 chip. Active low-pass filter circuit is shown in Fig. (4).

Cut-off frequency is $400 \mathrm{~Hz}$, capacitance takes $1 \mathrm{nf}$, the resistance of the calculation formula is:

$R=\frac{1}{2 \pi f c}=\frac{1}{2 \pi * 400 h z * 1 n f}=400 \Omega$

\subsubsection{Single-chip Computer Acquisition System Design}

Single-chip computer acquisition system includes the single chip microcomputer and the AD module. AD chip microcomputer completes $\mathrm{d} / \mathrm{a}$ conversion, then reads the value of the converted through RS-232 communication circuit, which reads the data sent to the PC for processing. 


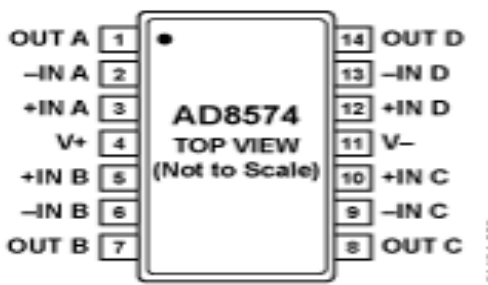

14-LEAD THIN SHRINK

SMALL OUTLINE

PACKAGE [TSSOP]

(RU-14)

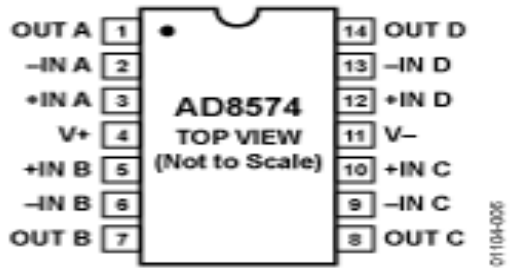

14-LEAD STANDARD

SMALL OUTLINE

PACKAGE [SOIC $N]$

NARROW BODY

(R-14)

Fig. (2). AD8574 pin figure.

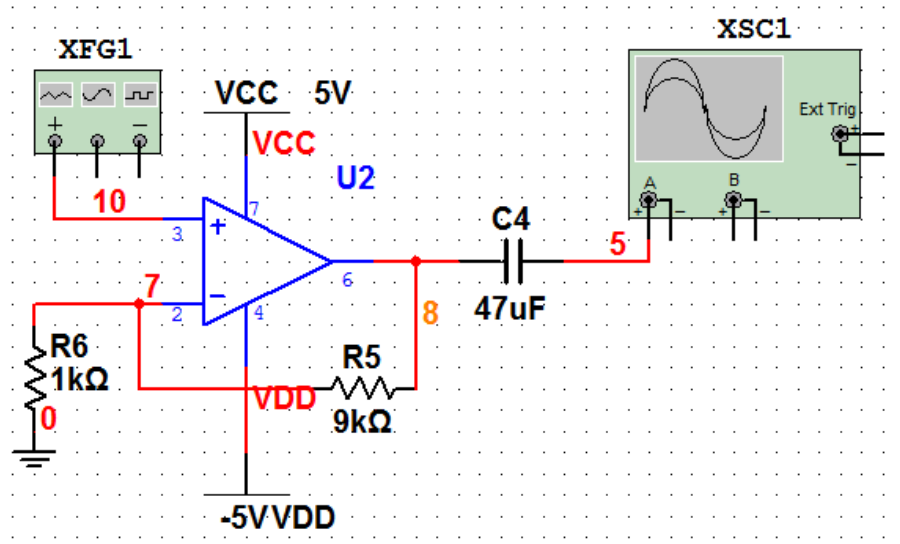

Fig. (3). The front-end amplifier circuit design.

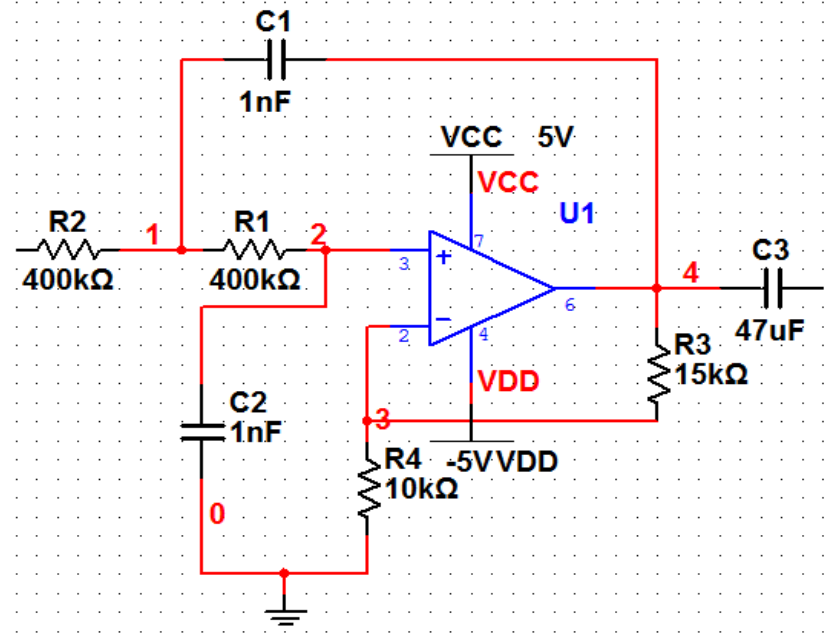

Fig. (4). Active low-pass filter circuit.

\section{D-A conversion}

Analog signals need to be converted into digital signals to identify for SCM or PC, so it needs d/a conversion circuit. Due to the frequency of the seismic signal is $20 \mathrm{hz}$ to $300 \mathrm{hz}$, the channel of each $\mathrm{d} / \mathrm{a}$ conversion sampling rate is at least $18.7 \mathrm{k}$ to collect at least ideal waveform. This system can detect four-channel microtremor signals at the same time, so based on the above request selected AD7856 as AD module of chip.

AD7856 is a high speed, low power consumption, $14 \mathrm{AD}$ converter, has the following features: 


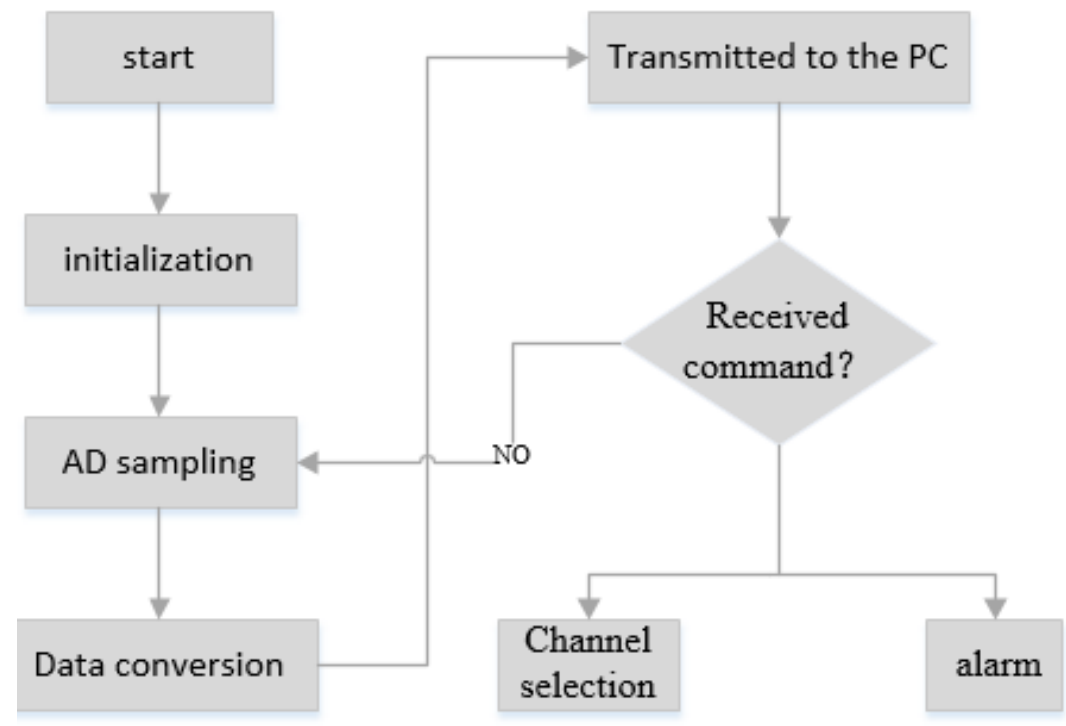

Fig. (5). Acquisition system flow chart.

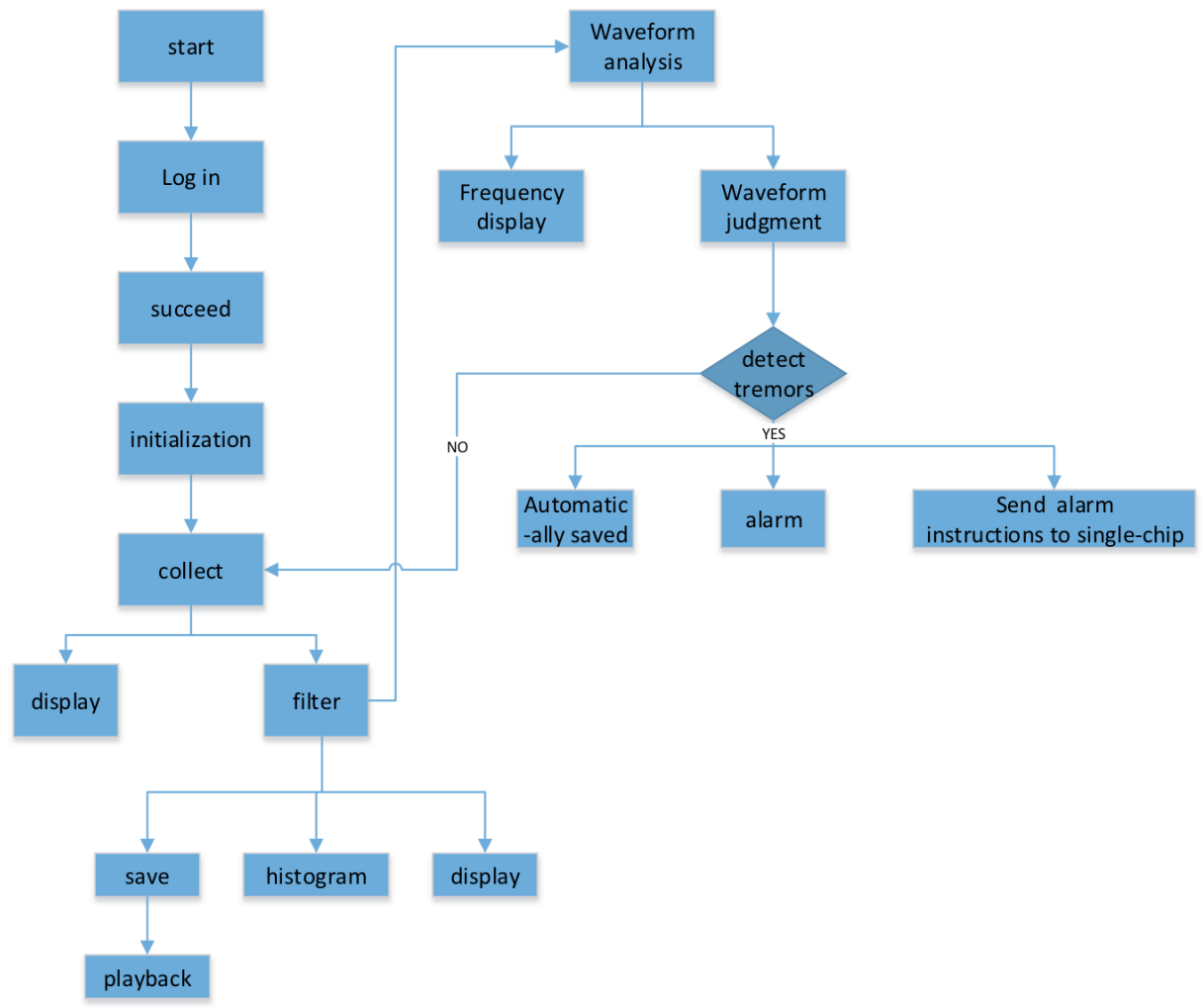

Fig. (6). Virtual instrument program flow chart.

Using $5 \mathrm{~V}$ single power supply.

Multi-channel: eight single-ended input or four pseudo differential input.

The throughput is up to $285 \mathrm{KSPS}$.

Low power consumption: $60 \mathrm{~mW}$ (typical).

Flexible serial interface: 8051 / SPI ${ }^{\circledR} /$ QSPI TM / $\mu$ P compatible.
Contains self-calibration and system calibration options to ensure that the operation precision is not affected by time and temperature.

2. Acquisition system flow chart

Compiles microcontroller program uses keil platform, program block diagram is shown in Fig. (5): 


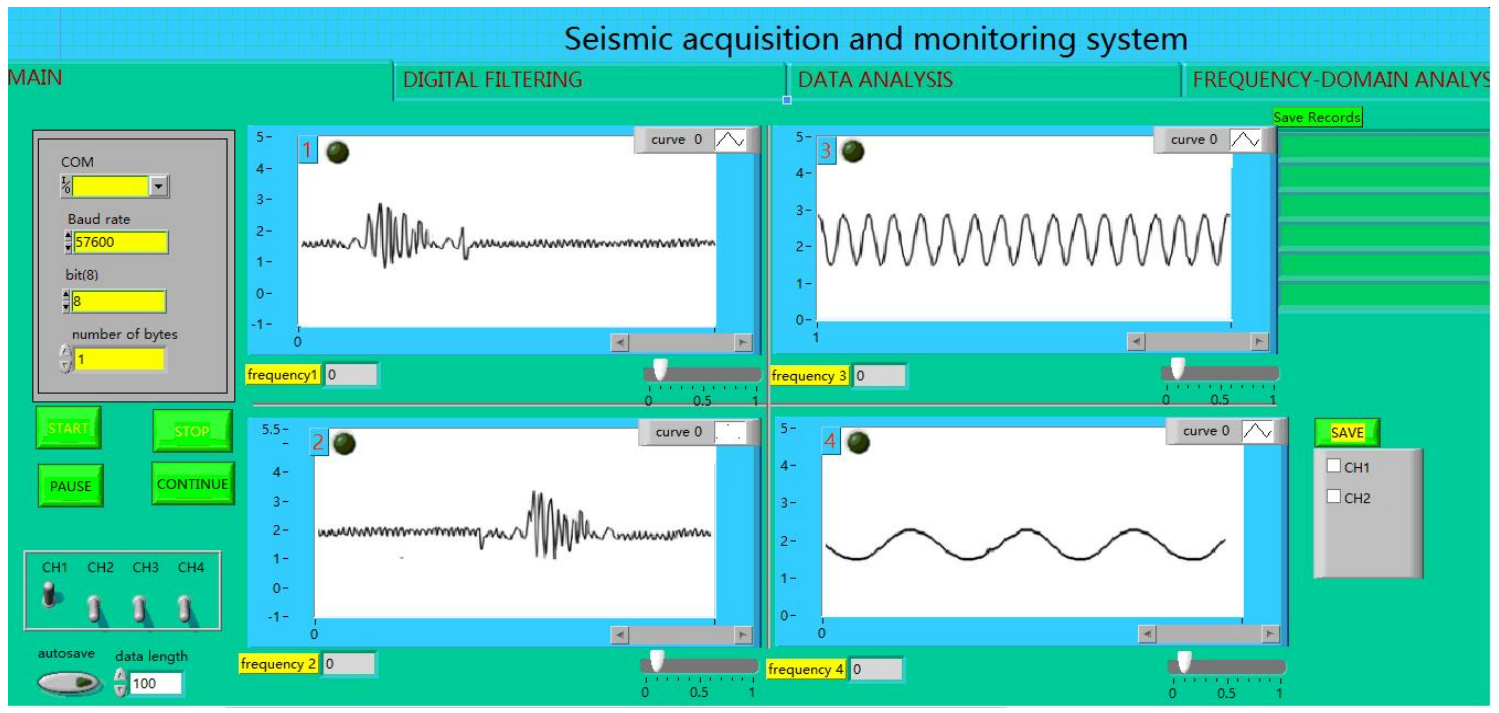

Fig. (7). The panels before receiving programs.

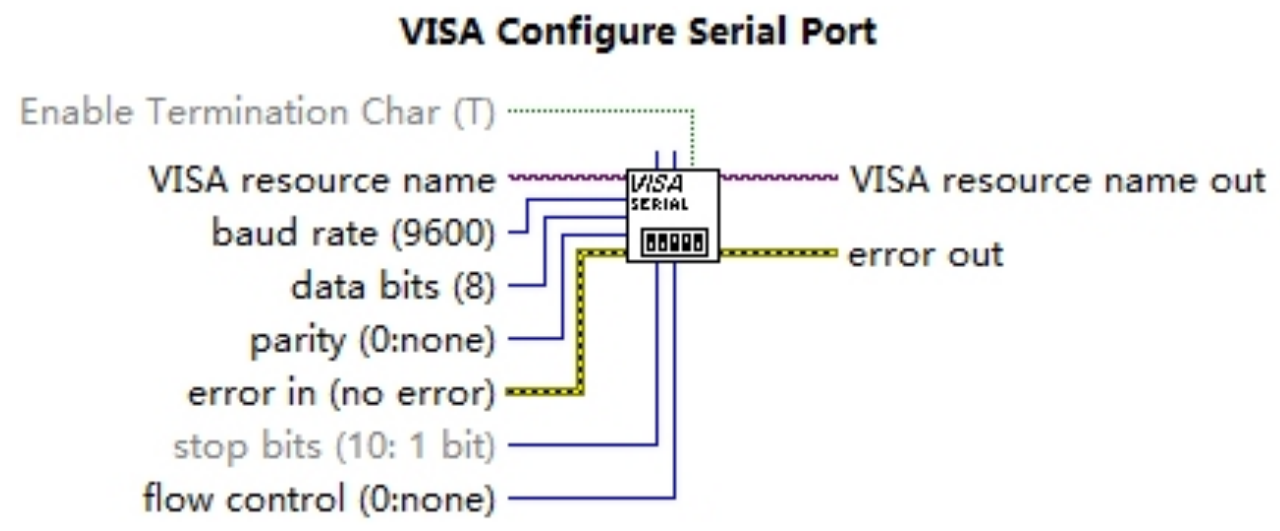

Fig. (8). VISA configure a serial port control.

\section{MICROSEISMIC MONITORING INTERFACE OF VIRTUAL INSTRUMENT}

\subsection{The System Software Framework as a Whole}

The software interface program block diagram of this system as shown in Fig. (6).

The subroutine of virtual instrument in the system design mainly includes the six modules, respectively is: VISA a serial port to send and receive module, data processing module, test module, frequency domain analysis, storage and playback module, log in $[9,10]$.

\subsection{A Serial Port Receiving and Sending Module of VI-} SA

Serial data receiving and sending part is crucial in the whole program. Its parameter is setted correctly or not, directly affect whether the back of the analysis, processing, display, and other functions can be fulfilled or not.

This module includes both receiving and sending.
1. The receiving application

The receiving application front panel as shown in the Fig. (7):

To receive the single-chip computer via a Serial Port to send rightly it needs to set some configuration for VISA Con Figure Serial Port control. The VISA configure serial port vi is shown in Fig. (8).

The programming process is as follows:

(1). It utilizes VISA Configure Serial Port to make a Serial Port initialization, The instruments are connected to the PC via a Serial Port COM9, VISA ResourceName sets as ASRL1:INSTR, which is consistent with the parameters of single chip, and transmission rate is $115200 \mathrm{BPS}$, it can send a 115200 - bit data per second. The basic set is 8 bits of data, 1 stop bit, and white parity bit.

(2). The nodes of VISA Write send a command to the single chip microcomputer, and the nodes of VISA Read read the data from the microcontroller data register. 


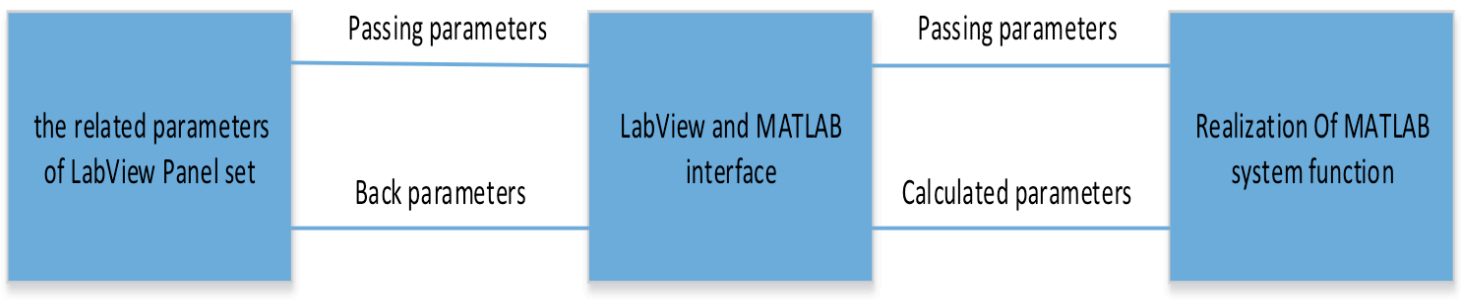

Fig. (9). The principle block diagram of wavelet de-noising.

(3). The VISA Read read data to displaye in the waveform chart. Using the event structure between each node set, the front panel and block diagram synchronization perform synchronous, which save the CPU resources.

\section{Sending the application}

In order to better control the acquisition process of slave computer, it needs upper computer to control the superior collection function of single chip microcomputer with real time. The channel selection function of this system design can send commands to the microcontroller ways for controing channel on or off, when the channel selection switch four changes, it sends instructions to the single-chip microcomputer system to execute the action of corresponding channel. In order to simplify the control process of program design of the four switch respectively as four binary number four bits, such as open channel 1,2, channel 3, 4 close then four binary number is 1100 , the number sent to the singlechip microcomputer to determine the channels open or close.

The programming process is:

1. The channel switch value converted to a binary number 0 or 1 .

2. The four binary convert to hexadecimal number according to the order.

3. Sending the hexadecimal number mcu to judge the four channels open and close.

Due to the Hexadecimal number, which is made by four channels ,is 0 to $\mathrm{F}$. The upper machine arranged according to MCU receives the instructions of channels to decide opening circuit, the process and the corresponding processing.

\subsection{Data Processing Module}

1. The power frequency filtering

Compared with the traditional analog filters, digital filter can use the software programming, high stability, predictable, and will have error because of the influence of temperature, humidity, etc. The LabVIEW platform provides a powerful tool for digital filter. When the microseismic monitoring circuit go through the power line, micro vibration sensor cable is induced voltage of $50 \mathrm{~Hz}$, formation of $50 \mathrm{~Hz}$ sinusoidal interference waves. Therefore in the LabVIEW platform, the system introduced band-stop filter to filter out the interference of power frequency $50 \mathrm{~Hz}$.

\section{Wavelet de-noising}

The design principle is: the LabVIEW contains noise signal, which is obtained by data collection, according to the instrument panel set up by the de-noising parameters, Passing the parameters to LabVIEW and MATLAB interface to the corresponding MATLAB functions, then the powerful MATLAB toolbox can realize the function of the wavelet function to complete the signal analysis and processing. Finally, the de-noising results displayed in the instrument panel. The principle block diagram is shown in Fig. (9):

\subsection{Storage Module}

To facilitate the subsequent processing and playback, the system can keep the waveform data to collecte to the PC. Making full use of the capability of the LabVIEW on file operations and the advantages of PC hard disk storage space.

The user can also save the current hoping waveform processing, select to save the channel, then the save will be based on "the current system time + channel number" save file to the specified folder, program diagram as shown in Fig. (10):

\section{WAVELET DE-NOISING ALGORITHM RE- SEARCH}

The concept of wavelet transform is first introduced by the French geologists Morlet $\mathrm{J}$ and GrossmannA analysis processing seismic data in the $70 \mathrm{~s}$, and successfully applied to the analysis of the seismic signal. Later, the famous French mathematician MeyerY theoretically make a series of research on wavelet. Ameodo and Grasseau applied wavelet analysis to mix pool dynamics and fractal theory to study turbulent and fractal growth phenomenon in 1988. In 1990, chui constructed single orthogonal wavelet function, which is based on spline function. Qin made clearance application of wavelet transform for geomorphologic mapping pretreatment in 1992, satisfactory results were obtained. Some domestic scholars view wavelet analysis as a great application prospect area of research $[10,11]$.

Using the multi-resolution analysis of wavelet analysis can be focused to any details of signal time and frequency domain processing, Therefore, it's very suitable for normal 


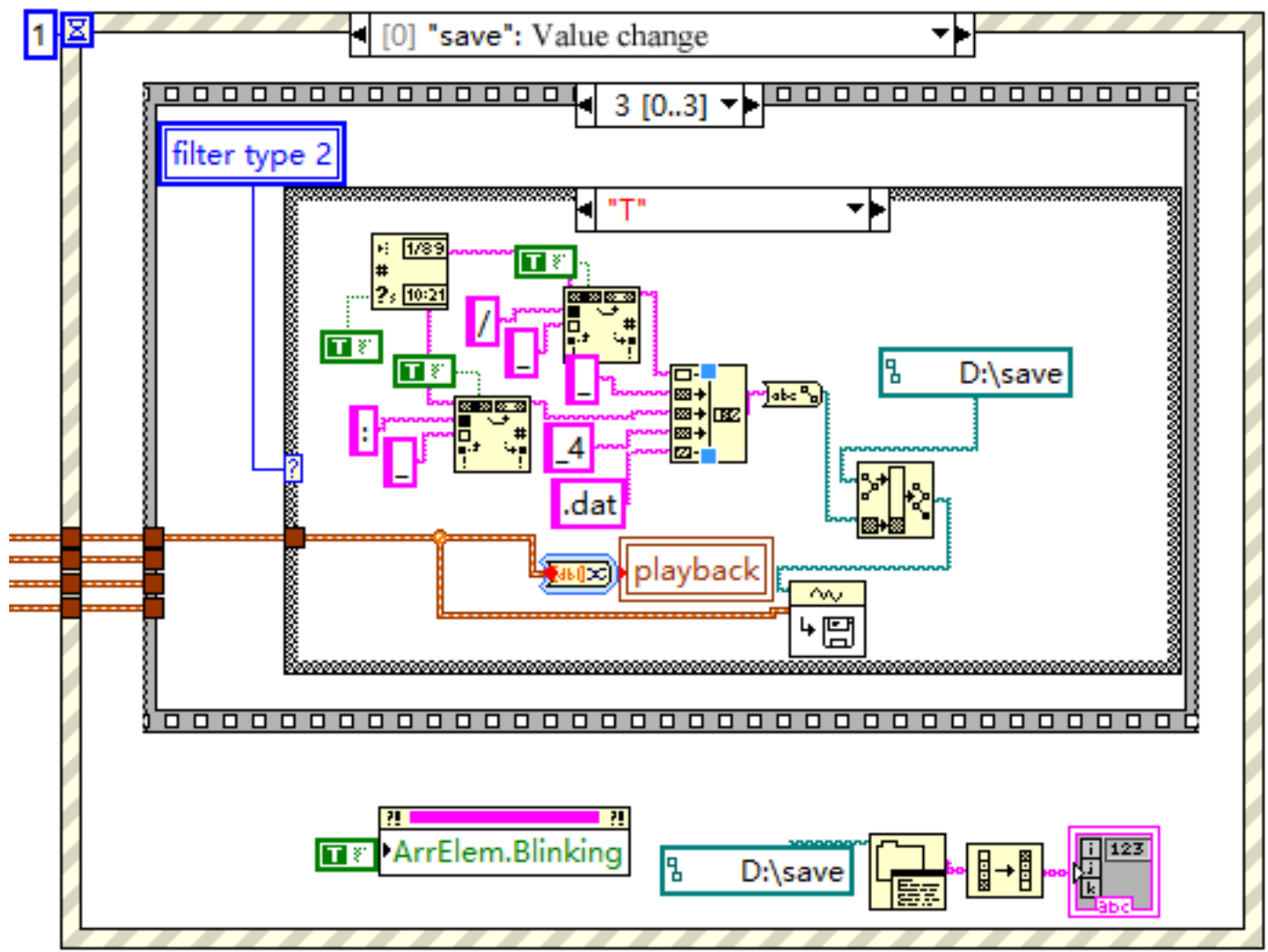

Fig. (10). Storage module Saving program default path for the D: \ waveform preservation.

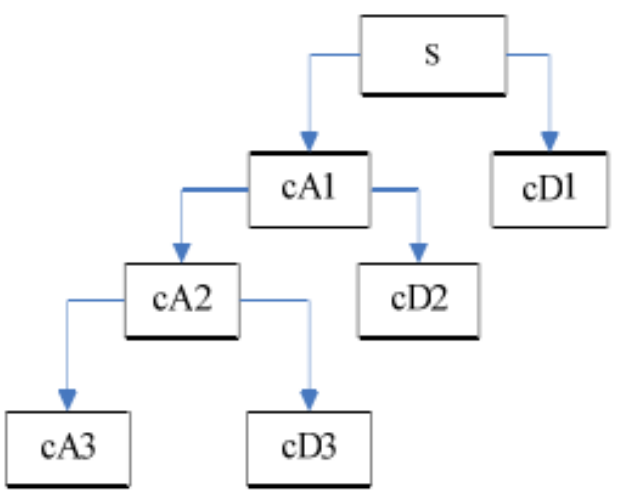

Fig. (11). Wavelet decomposition structure.

signal detection in the midst of transient abnormal phenomenon and shows its composition, especially the nonlinear wavelet noise separation method proposed in recent years, recovering the original signal waveforms from the mixed with strong noise signal obtained obvious effect. Also, this article use the method of wavelet transform the for first time to make preliminary research about the sensor signals with noise signal method provides an update.

If the window function $\phi(t) \in L^{2}(I R)$, meeting admissibility conditions:

$$
C_{\phi}=\int_{-\infty}^{+\infty} \frac{|\bar{\phi}(w)|^{2}}{|w|} d w<\infty
$$

Continuous wavelet transform of the function $f(t) \in L^{2}(I R)$ is defined as:

$$
(C W T f)(a, b)=|a|^{-1 / 2} \int_{-\infty}^{+\infty} f(t) \overline{\left(\frac{t-b}{a}\right)} d t=\left(f, \phi_{a, b}\right)
$$

Among them, the function is 


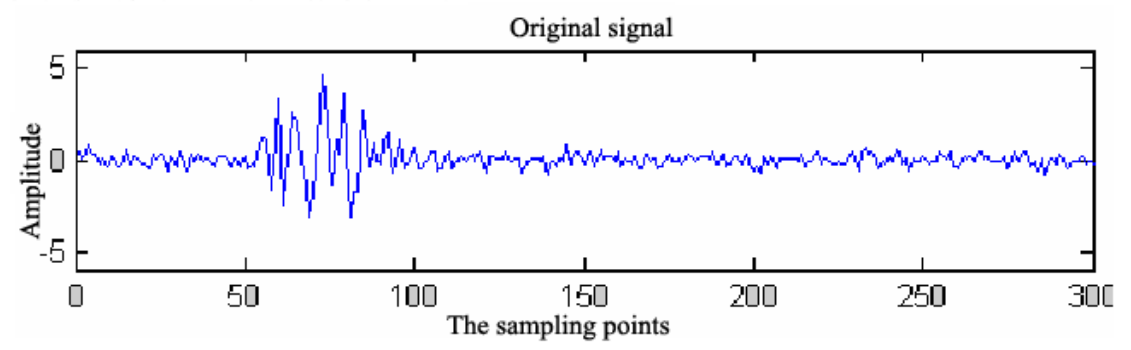

Fig. (12). Seismic waveform figure before signal de-noising.

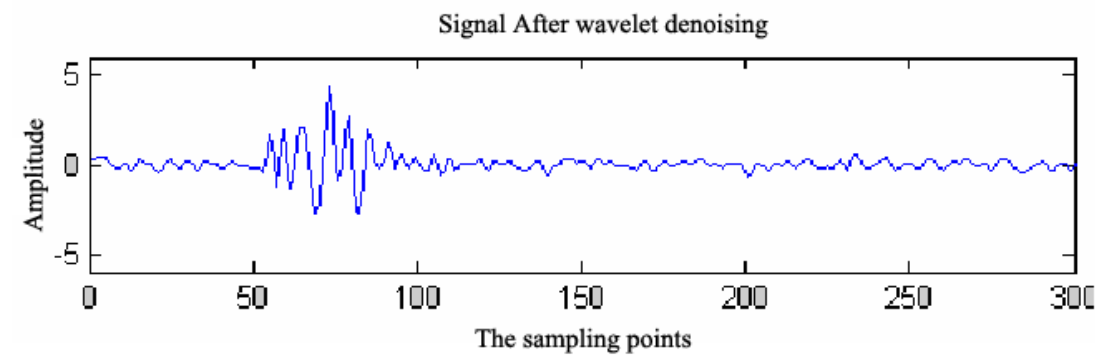

Fig. (13). Seismic waveform figure after signal de-noising.

$\phi_{a, b}(t)=|a|^{-1 / 2} \phi\left(\frac{t-b}{a}\right), a \in R, a>0, b \in R$

As wavelet function or little wave. It is gotten by a function by $\varphi(t)$ go through different time scale expansion and time shift. $\varphi(t)$ is known as the mother wavelet, a is time axis dimension scaling parameters, $b$ is the time shift parameters, different values of wavelet along the time axis to move to a different position. Signal $f(t)$ on $\varphi(t)$ of the continuous wavelet transform is the decomposed or projection of signal on the basis function.

\subsection{The Wavelet De-noising Theory}

Using wavelet analysis one dimensional signal denoising processing is one of the important application of wavelet analysis. A model of a one dimensional signal containing noise can be represented as the following form:

$s(i)=f(i)+\sigma \cdot e(i) \quad i=0, \ldots, n-1$

f(i) is true signal, e (i) for noise, s (i) for signals with noise. In the actual engineering, the useful signal is usually characterized by low frequency signals or more smoothly, and noise signal is usually characterized by high frequency signal. So the de-noising process can be treated as follows: First, the signal wavelet decompose, such as 3 layers of decomposition, decomposition process is shown in Fig. (1), Noise is often included in $\mathrm{cD} 1, \mathrm{cD} 2, \mathrm{cD} 3$, therefore, it can processe wavelet coefficient in the form of threshold, then the signal reconstruction can achieve the goal of de-noising. The purpose of signal s(i) de-noising is suppressing the noise in the signal, thus restoring the true signal $f(i)$ in the $s$ (i). The wavelet decomposition structure is shown in Fig. (11).

\subsection{Seismic Signal Analysis of the Wavelet De-noising}

Actual monitoring to the seismic signal is the process of sudden short, it is an obvious non-stationary signal. If you want to process and analyze its characteristic information, using Fourier transform and short-time Fourier transform method obviously appeared insufficient. At the same time, in the seismic signal, noise generally concentrated in the high frequency part, signal is concentrated in the low frequency part, and wavelet transform analysis signals in time and frequency domain at the same time, it can effectively distinguish between mutations in parts of the signal and noise, so as to realize the signal de-noising and reduction. In this paper, a set of real-time acquisition of microseismic signal wavelet de-noising, the results is shown in Fig. (12) and Fig. (13).

By the experimental results, it can be seen that the original signal contains a lot of noise and burr. After the reconstruction of the wavelet transform to get the signal, in addition to wipe off the obvious burr phenomenon and improve the SNR of signal. Compared with the traditional Fourier transform, wavelet transform can effectively distinguish between mutation part of microseismic signal and noise, so as to realize the signal de-noising. Using the wavelet analysis is a good way to keep the noise characteristic of the useful signal $[12,13]$.

\section{CONCLUSION}

We put forward a method of seismic signal acquisition and monitoring, the signal conditioning circuit and singlechip microcomputer system for signal collection, virtual instrument interface of the system software development, and using wavelet analysis in signal de-noising and refactoring. 
The system possesses friendly interface, good real-time performance, low cost, and has a broad application prospect in the safety monitoring of mine. System adopts LabVIEW development platform, and has good extensibility, it provides a good platform for the perfection of the system and development and design.

\section{CONFLICT OF INTEREST}

The authors confirm that this article content has no conflicts of interest.

\section{ACKNOWLEDGEMENTS}

This work is partially supported by JiangXi Science Foundation for Youths grants, China (No.GJJ12369), by Jiangxi University of Science and Technology grant, China (No.jxxj11029 and No.jxxj11033).

\section{REFERENCES}

[1] L. Liu, X. Liu, and G. Mei, "The coal mine safety in production accident cause factor analysis in China," China's Coal, vol. 45, no. 10, pp. 90-92, 2008.

[2] X. Wang, X. Dong, and L. Cao, "New technology of mine disaster warning and rescue system," Coal Mine Safety, vol. 38, no. 7, pp. 93-96, 2007.

[3] C. Li, "Single Chip Microcomputer Principle and Interface Technology, ( $3^{\text {rd }}$ ed)", Beijing University of Aeronautics and Astronautics Press, China, pp. 118-120, 2010.
[4] J. Zhan, Z. Ke, and X. Cao, "Characteristics microseismic monitoring experiment research of geological disasters," Survey Section, vol. 19 , no. 1, pp. 61-64, 2002 .

[5] Y. Zhang, "Rock acoustic emission and microseismic monitoring positioning technology and its applications," Blasting, vol. 8, no. 1, pp. 58-61, 2002.

[6] D. Wang, and Q. Xia, "Real-time microseismic monitoring system based on DSP technology," Micro Computer and Application, vol. 3, pp. 15-18, 2005.

[7] L. Dou, and X. He, Rock-Burst Prevention Theory and Technology, China University of Mining Press: Xuzho, 2001.

[8] L. Liu, F. Xia, and Z. Wang, Deployment issues in wireless sensor networks, In: Proceedings of MSN 2005, Lecture Notes in Computer Science, 2005, pp. 239-248.

[9] Y.P. Zhangs, "Novell nodel for ProPagation loss predietion intulinels," IEEE Trallson Vehicular Teehnology, vol. 52, no. 5, 2003.

[10] R. Zhang, D. Lin, S. Su, J. Wu, and L. Qiao, "Real-time microseismic monitoring system based on virtual instrument," Metal Mines, vol. 3, pp. 122-125, 2011.

[11] Q. Zhu, F. Jiang, Y. Yin, "Classification of mine microseismic events based on wavelet-fractal method and pattern recognition," Chinese Journal of Geotechnical Engineering, vol. 38, no. 3, pp. 2036-2042, 2012.

[12] Q. Xie, Z. Long, and M. Zhong, “Application of wavelet packet and fractal combination technology in blasting vibration signal analysis", Journal of Vibration and Shock, China, vol. 30, no. 1, pp. 11-15, 2011.

[13] Y. Hui, and Z. Feng, "Wavelet strategies and evaluation indicator in time sries data denoising," Geomatics and Information Science of Wuhan University, vol. 37, vol. 11, pp. 374-377, 2012.

Received: September 16, 2014

(C) Zeng et al.; Licensee Bentham Open.

This is an open access article licensed under the terms of the Creative Commons Attribution Non-Commercial License (http://creativecommons.org/licenses/by-nc/3.0/) which permits unrestricted, non-commercial use, distribution and reproduction in any medium, provided the work is properly cited. 